\title{
Comparison of Routing Protocols in Wireless Sensor Networks for Monitoring Applications
}

\author{
Mohamed I. Gaber \\ Nuclear Research Center \\ Atomic Energy Authority \\ Cairo, Egypt
}

\author{
Imbaby I. Mahmoud, Osama \\ Seddik \\ Nuclear Research Center \\ Atomic Energy Authority \\ Cairo, Egypt
}

\author{
Abdelhalim Zekry \\ Faculty of Engineering \\ Ain Shams University \\ Cairo, Egypt
}

\begin{abstract}
In recent years, evaluation and development of the routing protocols in wireless sensor networks (WSNs) are very important and attractive research topic especially for monitoring applications. Because of, the difficulties of studying WSNs routing protocols in real implementation which takes a lot of time and it can be very expensive, using a suitable simulator become a common trend in such evaluation. This paper presents a systematic performance study of three routing protocols, Ad hoc On Demand Distance Vector (AODV), Dynamic Source Routing (DSR), and Optimized Link State Routing (OLSR) protocols for WSNs by proposing a simulation model that targeted to the sensor networks with mobile sensor nodes and single sink as it is often seen in many monitoring applications such as military, agriculture, medical, transport, industry, etc to monitor physical environments. The performance study of WSNs routing protocols is analyzed by comparing important metrics like the end-to-end delay, total packets dropped, load, routing overhead, route discovery time, and number of hops per route in the Network under the same random waypoint mobility model for the three protocols. These routing protocols are implemented and simulated using OPNET Modeler simulator. Theoretical analysis and simulation results show that both AODV and DSR protocols have identical on demand behavior but with performance differentials resulted from the differences in protocol mechanics. In addition to, they are suffering from higher end to end delay compared to the Optimized Link State Routing (OLSR) protocol. The results obtained may be useful for implementation of wireless sensor networks for many monitoring and control applications.
\end{abstract}

\section{General Terms}

Wireless Sensor Networks, Routing Protocols, OPNET

Modeler, Monitoring Applications, Mobility Model

\section{Keywords}

WSNs, Routing, Monitoring, AODV, DSR, OLSR, OPNET

\section{INTRODUCTION}

Wireless Sensor Networks (WSNs) represent the next technological revolution in our world which differ from other wireless networks through its capability of interaction with the environment for monitoring. These networks can be defined as wireless networks consist of a large number of tiny, low power, cheap, smart and multifunctional sensor nodes that are deployed in a sensing region of many emerging areas without requirement of any existing infrastructure. These sensor nodes equipped with sensing unit to sense the events, embedded micro-processor for data processing, battery as power supply unit, and radio transceiver unit can communicate sensor nodes with each other to monitor real world physical or environmental conditions such as temperature, vibration, pressure, motion, radiation, etc. These nodes can cooperatively pass the sensed data to a main location called base station or sink that are located near or inside the sensing area. Afterwards, pass the sensed data through the network gateway to the operator station where the data can be observed and analysed. Sink or base station acts like an interface between the operator and the sensors network, reliable and proficiency routing of data packets are very important goals for data transmission in Wireless Sensor Networks (WSNs).

\section{RELATED WORK}

In recent years, we found several research papers in the literature related to studying and comparison of routing protocols in WSNs. For example, the author of [1] shows the performance evaluation of AODV and DSDV protocols in WSN by comparing them according to the end to end delay, packet delivery fraction, and throughput in different environment using the same IEEE 802.11 Media Access Control (MAC) layer in all experiments. The overall observation shows better performance of AODV routing protocol in terms of packet delivery fraction and throughput but suffers from delay that similar to ours Simulation results. The authors of [2] Show the performance comparison of two on-demand routing protocols (AODV and DSR protocols) for ad hoc networks by using varying network load, mobility, and network size. The presented study shows that the two protocols share the on-demand behaviour, but with different routing mechanics and DSR was more effective at lower loads, while AODV was more effective at higher loads. Similar to ours Simulation results DSR protocol generated fewer overall routing packets. The authors of [3] discussed the importance role of mobility model in analysing the performance of routing protocols. They discussed various mobility models with our used random waypoint model, they also show the importance of the two key parameters $\mathrm{V}$ (maximum allowable velocity) and $\mathrm{T}$ (pause time) in determine the mobility behaviour of mobile nodes. The authors of [4][5] evaluated the performance of on-demand routing protocols and their algorithms with comparison between important parameters such as delay, packets drop and packets delivery ratio for DSDV, AODV, and DSR routing protocols which use on-demand route discovery procedure, but with different routing mechanics. The results show that the delay of DSR is slightly less than DSDV\& AODV for increasing number of packets and DSR packets drop rate very low when compared to DSDV and AODV protocols. The work presented in [6] evaluated the performance of DSR routing protocol on WSN by studying the impact of the network size, network density, and the number of sources (data connections) on different performance metrics which are the average end-to-end delay, packet delivery fraction, routing overhead using NS-2 simulator. It is found that the DSR protocol has well performance in most of the tested scenarios with low latency and high packet delivery fraction. The work 
presented in [7] shows the comparison between AODV and DSR routing protocols by evaluating the ratio of received to $\operatorname{sent}(\mathrm{r} / \mathrm{s})$ with varying pause time for different Terrain areas. The results show that DSR routing protocol has very high average end-to-end delay and throughput in the case of comparison between AODV, DSDV, and DSR protocols, but in comparison between DSDV and AODV routing protocols, AODV performed better than DSDV in terms of bandwidth as AODV do not contain routing tables.

Paper organization is as follow; Section 3 surveys the implemented routing protocols and their algorithms in wireless sensor networks. Section $\mathbf{4}$ contains analysis of the network performance using network simulator (OPNET Modeler) with discussion of used mobility model, and describes the proposed radiation monitoring application depends on the proposed wireless sensor network simulation model with its parameters. Section $\mathbf{5}$ discusses analysis of the results obtained from the previous section. Finally section 6 concludes the work.

\section{IMPLEMENTED ROUTING PROTOCOLS DESCRIPTION}

Routing [8] is the process of establishing paths from a given source node to a given destination node with the ability of using intermediate nodes to reach the final destination. In WSNs, the sensor nodes can collaborate to collect the sensing task and send the sensed data to the sink node that sends queries to the sensor nodes in the sensing region to request the sensed data, the sink or base station node also serves as a gateway to outside networks through the Internet. It collects the sensed data from the sensor nodes, performs simple processing on the collected sensed data, then sends the processed data via the Internet to the operator station who requested it to be analysed [9] and sends the data to database station .Routing protocols [8] [10] are responsible for discovering routes from the source or sender to the intended destination. On the basis of route calibration process, routing protocols are categorized in three ways: reactive (On Demand), proactive (Table driven), and hybrid routing protocols. Reactive routing protocols establish routes on-demand only when actually needed to send data between a source and a destination node. Proactive routing protocols establish routes before they are actually needed where less delays are occurred to search for routes such as in reactive routing protocols, these proactive protocols are also often described as table-driven because the data routing mechanism based on the contents of established routing table that includes a list of destinations, but the disadvantages of proactive protocols are the heavy traffic or overhead routing traffic produced in building very large routing tables that can lead to increase the routing errors and dropped packets. The focus in this paper has been given to Ad Hoc On demand Distance Vector (AODV), Dynamic Source Routing (DSR) protocols that are chosen as examples to study the performance of reactive routing protocols and Optimized Link State Routing (OLSR) protocol that is chosen as an example to study the performance of proactive routing protocols.

\subsection{Ad hoc On-demand Distance Vector (AODV)}

It is an example of reactive routing protocols [8] that uses an on-demand approach to find and establish routes. It is essentially used for mobile ad-hoc networks, but in recent years it is widely used in WSNs. AODV maintains routes as long as they are needed by the source nodes and it is considered one of the best routing protocols that establish the shortest path and less consumed power. In AODV, each node behaves as a specialized router and routes are created ondemand only when actually needed to send data between a source and a destination node [11]. Whenever, a source node doesn't have a valid route in their routing table to the destination or a previously valid route expires, the source node initiates a route discovery process [2] [4] by broadcasting a route request (RREQ) packet to its neighbors. This packet contains the addresses of the source and the destination nodes, hop count field that sets to zero at the start of transmission, broadcast ID which is incremented with each RREQ sent and two sequence numbers used to ensure the freshness of information about the reverse route to the source. The neighbor nodes that do not know any information about the destination node in the network will send or rebroadcast the RREQ message to all its neighbors and so on. A duplicate RREQ (identified by its source address and broadcast ID) is discarded, but any neighbor node has a current route to the defined destination responds by sending a unicast route reply (RREP) message directly back to the neighbor node from which the RREQ packet was received. This neighbor node does the same behavior and then the route reply message travels back using reverse path of the route. On the basis of this process a path is recorded in the intermediate nodes. When a route reply message reaches to the source the route is ready and the source can start sending data packets. If many RREPs are received by the source, the route with the shortest hop count is chosen. AODV uses destination sequence numbers to get a network has fresh and loop free routes at all times. Also neighboring nodes exchange periodic HELLO messages between each other to monitor the state of their links. When the link along the route breaks by the movement of nodes, the intermediate node closer to the source node declaring that a broken link found and send a route error (RERR) packet upstream toward the source node, upon receiving an RERR packet, a source node can reinitiate the path discovery process by using route maintenance process [10][11].

\subsection{Dynamic Source Routing (DSR)}

It is another example of reactive routing protocols [8] [10] that behave the same route discovery and route maintenance procedures similar to AODV. In DSR protocol, the source node knows the complete route to the destination node from hop to another as it uses source routing technique where these routes are stored in a route cache [12]. The data packets carry the source route in the packet header, a node wishing to send a data packet will search in its route cache to see whether it already has a route to the destination. If there is no valid route in the cache, the sender initiates a route discovery procedure by flooding a route request packet through the network, each node receiving a RREQ packet inserts its own address into the request packet before re broadcasting it, unless it is the destination or it has a route to the destination in its route cache. Therefore, the node replies to the RREQ packet by a route reply (RREP) packet that is routed back to the original source. The RREQ builds up the path traversed across the network and the RREP routes itself back to the source by traversing this path backward. The route carried back by the RREP packet is cached at the source for future use [10]. Similar to AODV, if any link on a source route to a destination is broken, the source node is announced to initiate a route maintenance procedure based on a route error (RERR) messages. The source node removes any route using this link from its cache and a new route discovery process must be initiated by the source node if this route is still needed. The advantage of DSR protocol is that each data packet in DSR protocol conveys route information that allows intermediate 
nodes to add new routes to their own caches and can support of data routing in asymmetric links, in addition to DSR routing protocol has less routing traffic overheads with data packets because it uses source routing mechanism.

\subsection{Optimized Link State Routing (OLSR)}

It is an example of proactive routing protocols [10][13] which routes are already available in routing table so less route discovery time delay can be occurred. OLSR based on the link state and characterized by two types of control messages: the first type is the topology control messages that sent to all other nodes in the network, allowing them to obtain a complete topological map of the network and to allow determines paths to any destination node in the network. The second type is the HELLO messages that used to get the link status information by using neighbor sensing to identify its neighbors and determine information about its two-hop neighborhood. Only selected nodes called multipoint relays (MPRs) are used to broadcast topology information through the network during the flooding process instead of allowing each node to broadcast topology messages which happened due to classic flooding process shown in Figure 1 (a). MPRs used for reducing the overhead traffic in the network and reducing duplicate transmissions. In OLSR, a node selects a set of symmetric neighbor nodes as MPRs that called the MPR selector set [10]. Only MPRs forward messages to other nodes as shown in Figure 1 (b). Topology Control (TC) messages contain information about which MPR node selected in the network. TC messages also make the calculation of routing tables for each node in the network. The advantage of OLSR protocol is that it has less average end to end delay and wider delay distribution so it is suitable for time critical applications.

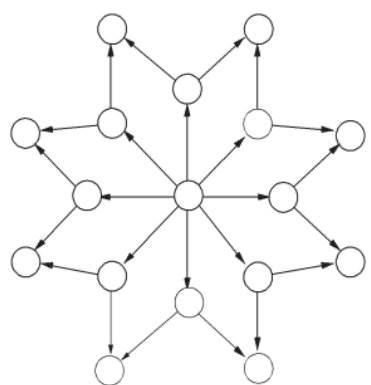

Figure 1: (a) Classic Flooding

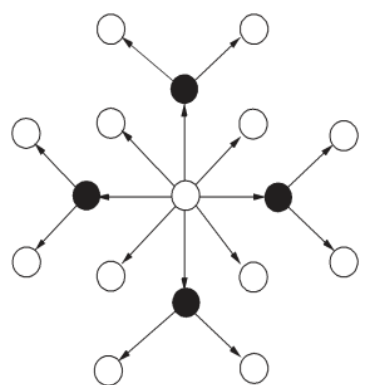

(b) MPRs based OLSR

\section{NETWORK PERFORMANCE ANALYSIS}

This section presents description about the used network simulator tool (OPNET Modeler), node mobility and the used random waypoint mobility model, performance analysis of the proposed network simulation model with their used parameters.

\subsection{The network Simulator Tool (OPNET Modeler)}

Simulation is an important tool to study the development of mobile ad hoc and sensor networks, it provides good environment to experiment and test routing protocols behavior. Optimized Network Engineering Tool (OPNET Modeler) [14] is a network simulator that provides solutions for managing networks and applications including networks engineering, operation, planning, and performance management. It used by a lot of institutes and researchers for modeling communication systems, devices, protocols, technologies, and to simulate the performance of these technologies in dynamic virtual network environment. Strength of OPNET Modeler in wireless networks simulation is the accurate modeling of the radio transmission. Both behavior and performance of a model can be analyzed by performing discrete event simulations. A Graphical User Interface (GUI) supports the configuration of the scenarios and the development of network models. The academic research in OPNET technology provides support for wireless protocols, Mobile Ad hoc network protocols, core network technologies, and used to simulate and evaluate the performance of Wireless Sensor Networks (WSN) architecture. In this paper, we focus on the technical scenarios which are an abstraction of a real world WSN application for radiation or environmental monitoring with two types of nodes: the sensor nodes attached to the detection areas and the sink node that collects the sensed information.

\subsection{Node Mobility and Random Waypoint Mobility Model}

In our work we supposed the random waypoint mobility Model [3][14] to evaluate the behavior of the routing protocols in wireless senor networks and describe the movement pattern of mobile nodes, and how their location, velocity change over time in order to test the protocols performance under actual conditions, especially including the movement of the mobile nodes. At the start of simulation time each mobile node waiting a specified pause-time[7] and after this time randomly selects one location as destination in the simulation field with constant velocity chosen uniformly and randomly from[0,Vmax], where the parameter Vmax is the maximum allowable velocity for every mobile node. After reaching its destination point, the mobile node waits again pause-time Tpause before choosing a new way point and speed. If Tpause $=0$, this leads to continuous mobility. After this duration, it again chooses another random destination in the simulation field and moves towards it. The whole process is repeated again and again until the simulation ends. The random waypoint mobility model is very widely used in simulation studies of mobile Ad hoc networks and sensor networks.

\subsection{The proposed Network Simulation Model}

In small sensor networks where the sensor nodes and the sink (gateway) are in near distance between them [9], it is suitable to use direct (single-hop) communication between all sensor nodes and the gateway. The sensor data may be collected in various plans. According to event-driven applications such as radiation detection, nodes only report their collected information when events of interest occur. In time-driven applications such as environmental monitoring, nodes propagate their collected sensor data periodically to a sink or gateway device. Finally, in query-driven applications, operator can request sensed data through the gateway device or sink that is responsible for sending a request of sensed data from sensor nodes when needed.

In this paper, we introduce the simulation model of AODV, DSR, and OLSR routing protocols in wireless sensor network for monitoring applications by proposing a simulation model in the network simulator (OPNET Modeler) [14] as shown in Figure 2 with simulation infrastructure contains the following items:

1- The wireless domain of random waypoint mobility model that consists of two elements (The first element is 
the mobile sensor nodes which cover our region of interest. The second element is the base station that has more processing, energy and communication capabilities than the other nodes and it can make a connection between the sensor network and other networks such as the Internet, the mobile networks. In addition to, it can collect sensed data and relay them to the database and operator station)

2- The server's tier that consists of (monitoring server or operator, database server, E-mail server) and all these servers connected to the sensor network by Internet.

3- The configuration definition tier that consists of (application definition node, profile definition node, mobility configuration node, receive group configuration node).

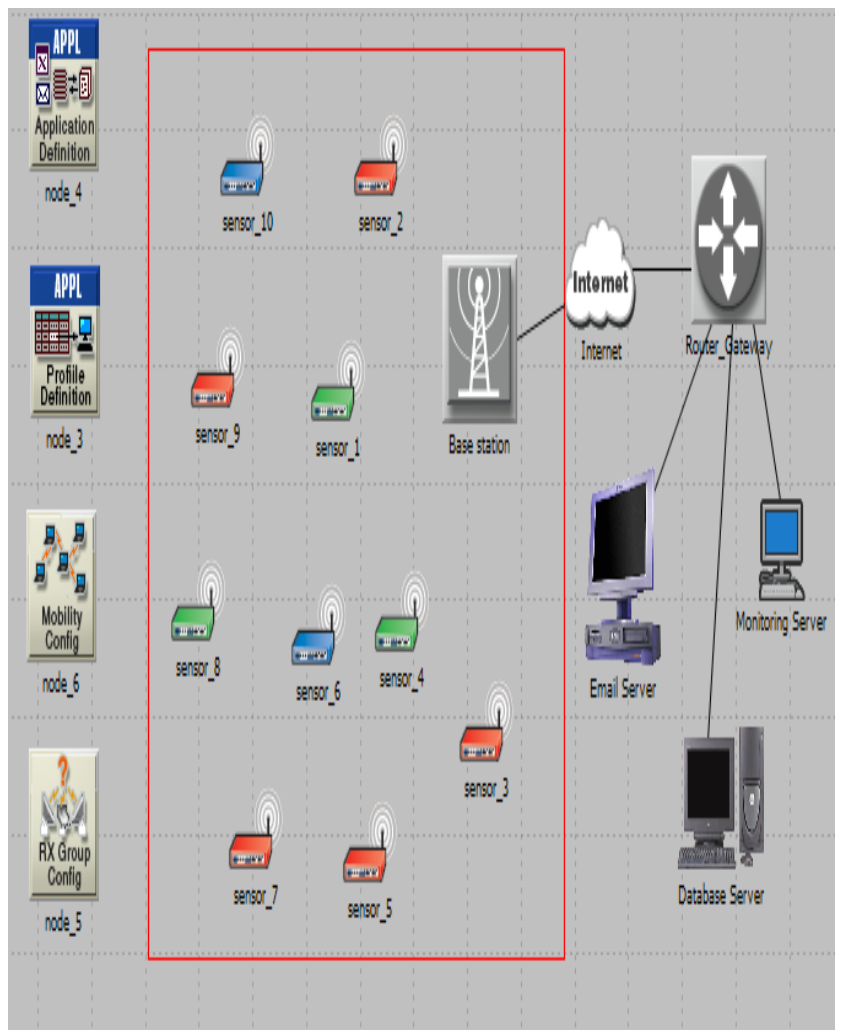

Figure 2: Proposed Simulation Model Layout

Table 1: Parameters of simulation

\begin{tabular}{|l|l|}
\hline Routing Protocols & AODV,DSR,OLSR \\
\hline Number of wireless sensor node & 10 nodes \\
\hline Movement space & $1000 \mathrm{~m} * 1000 \mathrm{~m}$ \\
\hline Distance Threshold(meters) & 500 meters \\
\hline Maximum speed & $100 \mathrm{~m} / \mathrm{s}$ \\
\hline Maximum pause time & $100 \mathrm{sec}$ \\
\hline Transmission power & $0.005 \mathrm{~W}$ \\
\hline Packet size & $512 \mathrm{bytes}$ \\
\hline
\end{tabular}

\begin{tabular}{|l|l|}
\hline Simulation time & $1200 \mathrm{sec}$ \\
\hline Antenna Model & Omni Antenna \\
\hline MAC type & IEEE $802.11 \mathrm{~b}$ \\
\hline Data rate & $11 \mathrm{Mbps}$ \\
\hline Mobility model & $\begin{array}{l}\text { Random waypoint } \\
\text { mobility model }\end{array}$ \\
\hline
\end{tabular}

\subsection{The proposed Radiation Monitoring System}

An important application of WSNs is the monitoring of environmental parameters and critical conditions. The presented application example can be applied to several WSN deployment scenarios. With the advancements in production of small, accurate, low power sensors, it is becoming more and more possible to deploy a WSN for continuous monitoring of radiation levels in nuclear regions. The WSN would report the radiation levels, and the critical measurements to be available to the operators via dedicated websites, mobile applications, etc. In addition, the stored measurements can be made available to expert environmental scientists to analyze and access the information in order to submit recommendations to the relevant authorities in order to take appropriate actions. The system model for radiation monitoring is displayed in Figure 2 where it can be applied to many monitoring applications. The Base Station (BS) covers a certain area of interest, several Sensor Nodes (SNs) are deployed to monitor environmental parameters such as radiation dose rate monitoring [16]. In this example, we presented a description of the proposed system architecture for radiation monitoring and described the role of the $\mathrm{SNs}$ where the presented routing protocols can be applied.

\section{SIMULATION RESULTS AND DISSCUSSION}

The performance metrics that under considerations are:

\subsection{Average end to end Packet Delay}

One of the most important goals in the sensor networks is to reduce the time delay because the least end to end delay gives better application performance; end-to-end packet delay is defined as the time elapsed between the creation of the packet at its source node and the time which the packet is received at the destination node [15]. As shown in figure 3 the global statistics for end to end delay of three routing protocols (AODV, DSR, and OLSR) in seconds with the simulation time in minute. At the start of simulation initiate pause time $100 \mathrm{sec}$ for random waypoint mobility. It's clear that both AODV and DSR protocols have the same on demand behavior where DSR delay is higher than AODV delay only in the start of packets transmission when the nodes establish the route during the route discovery procedure and decrease to lower than AODV delay during the rest simulation time, on the other side the OLSR delay is very low at the start and throughout the simulation time and takes minimal delay with wider delay distribution for determining the route to be taken because OLSR protocol has proactive routing characteristic which stores and updates its routes. When the route is needed, it presents the route immediately without any initial delay. Fewer delay of OLSR protocol is very useful especially for time critical traffic applications. 


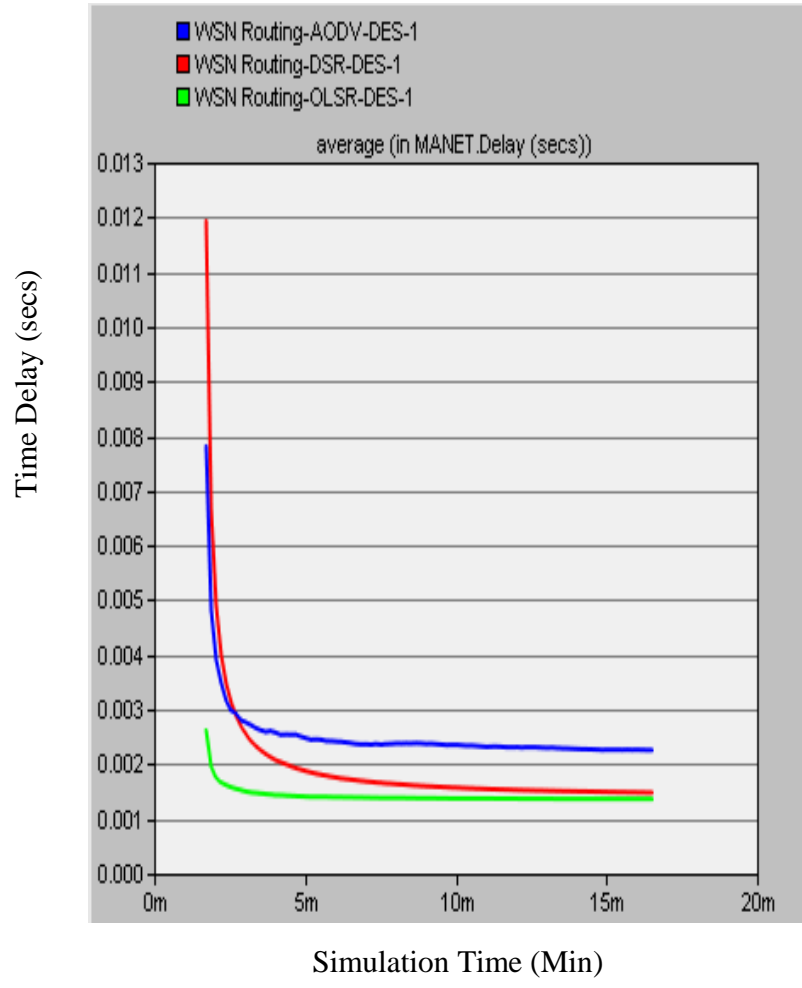

Figure 3: End to end delay for (AODV, DSR, and OLSR)

\subsection{Load}

This statistic represents the total load (in bits/sec) submitted to wireless LAN layers by all higher layers in all nodes of the network. If the load increased, it would create more traffic in the network that can decrease the speed of packets and increase the collision of control packets with more dropped packets. As shown in figure 4 DSR protocol has the lowest load but AODV protocol has the largest load. This result can approve that the least total load can produce fewer dropped packets in the network such as in the state of DSR protocol.

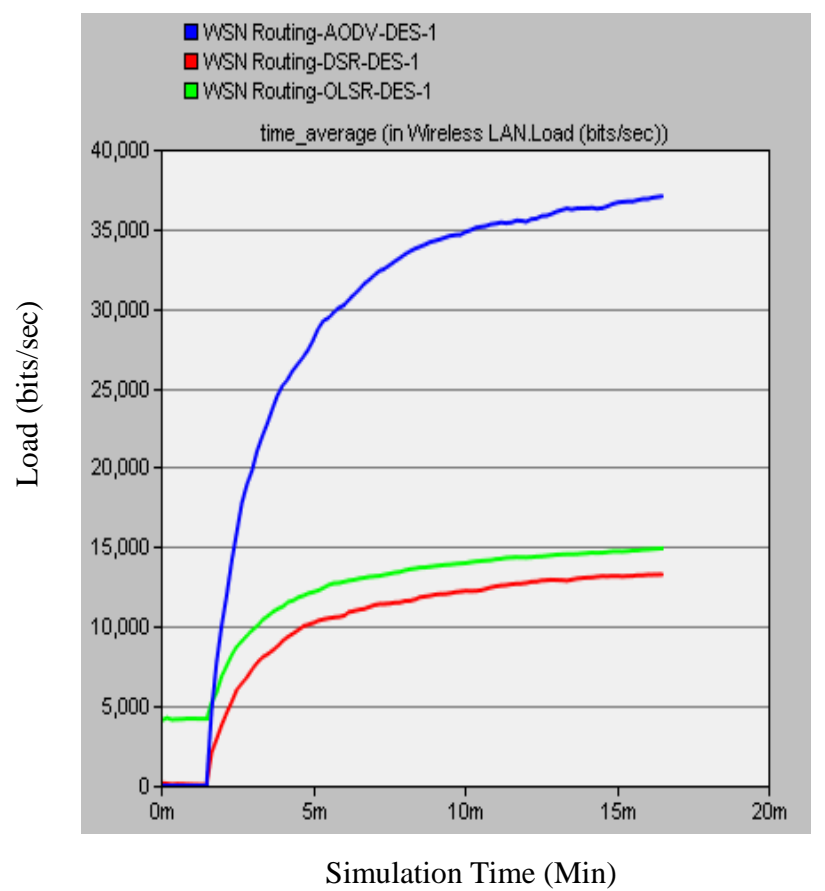

Figure 4: Load (bits/Sec) for (AODV, OLSR and DSR)

\subsection{Total Packets Dropped}

The total packets dropped can be defined as when no route is found to the destination, the node drops the packets queued to the destination. The statistics shown in figure 5 represent the total number of application packets discarded by all nodes in the network. This discard because of one of the following reasons: packet collisions and routing loop. As shown in Figure 5 DSR protocol has less dropped packets because of the defined routes in the header of packets give chance to route the packets correctly. But, as result to the flooding process in route discovery procedure, we find that the dropped packets increased in AODV protocol.

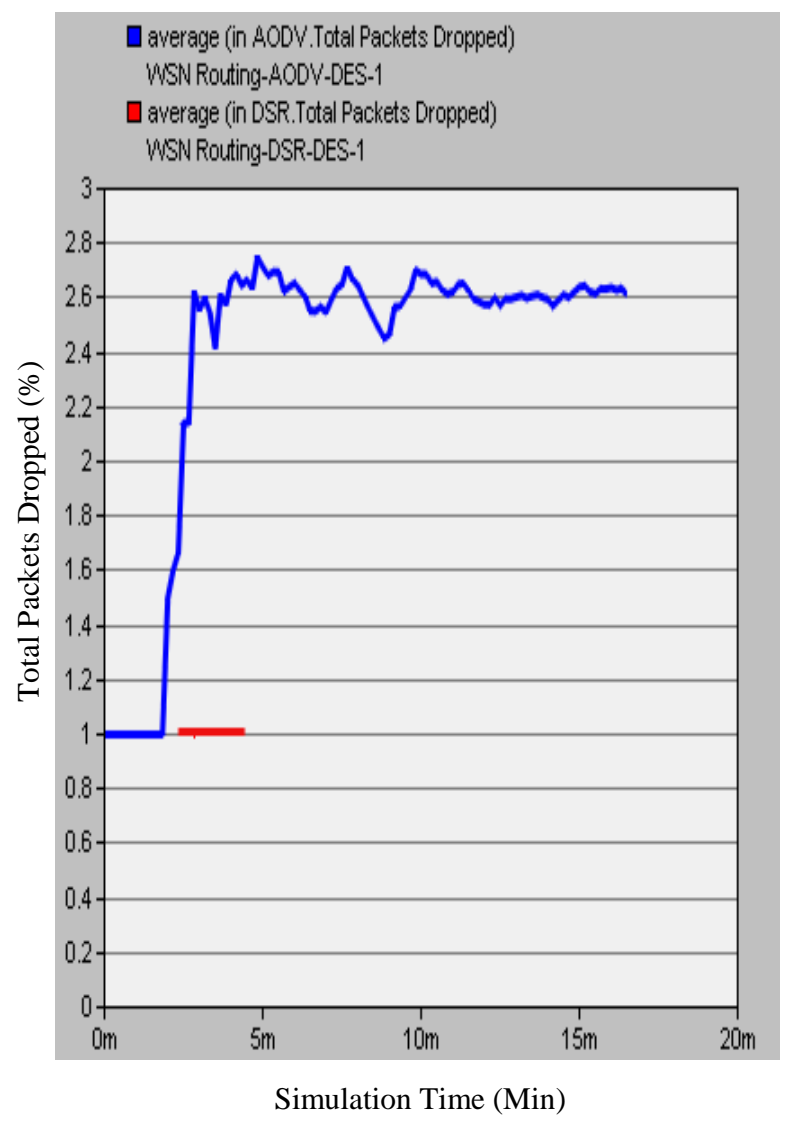

Figure 5: Total packets dropped (AODV and DSR)

\subsection{Routing Overhead}

Routing protocols send control packets to find routes. so, routing overhead can be defined as the total routing traffic sent (in bits/sec) by all nodes in the network during simulation. This metric is used to measure the efficiency of routing protocols as the largest overhead will waste more bandwidth. In Figure 6 we observed that routing overhead of reactive protocols increased at the start of simulation time in routes establishment process and increment of hello messages lead to waste more bandwidth. Intermediate nodes in DSR routing protocol are able to utilize the route cache information efficiently to reduce the control overhead. On the other hand, if we look at the behavior of OLSR that gives a consistent nature of routing overhead due to its proactive routing nature which paths to all nodes are already defined and calculated. The routing overhead created at the network resulted from the periodic updates of routing information for updating the routing tables which is slightly low. 


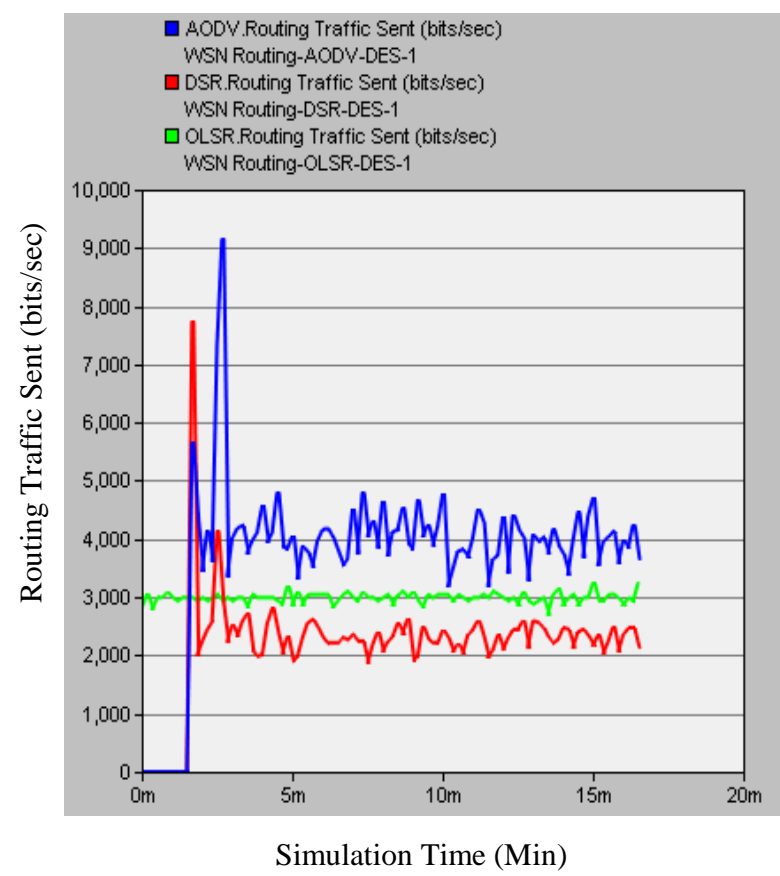

Figure 6: Routing overhead for (AODV, DSR, and OLSR)

\subsection{No of Hops per Route}

Number of hops per route represents the number of hops in each route to every destination in the route table of all nodes in the network. As shown in figure 7 we find that AODV protocol produces a steady one hop per route. But, the DSR Routing protocol produces approximates 2 hops per route. This indicates that AODV routing is offering one main route to the destination and DSR routing is offering multiple routes to the destination and can increase the opportunities of successful data transmission with minimum transmission power.

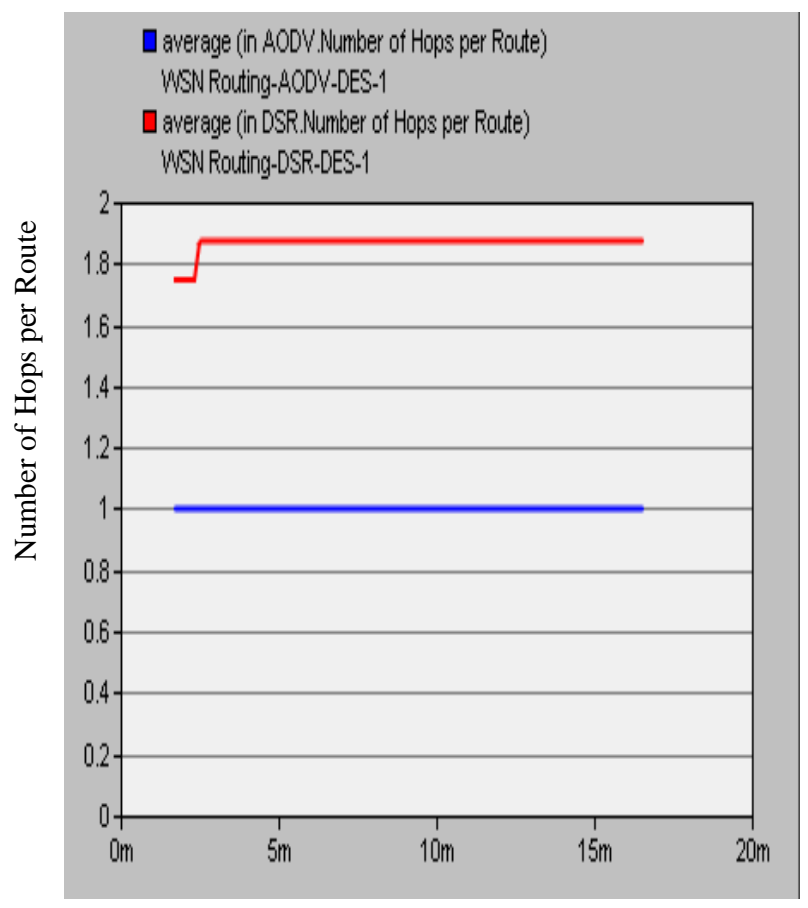

Simulation Time (Min)

Figure 7: No of hops per route for (AODV, DSR)

\subsection{OLSR Multi point Relay Status}

The OLSR node Multi point Relay (MPR) status is a good starting point to generate and forward the Topology Control (TC) messages in OLSR routing protocol. It indicates if a node selected as MPR during a simulation or not. It produces usually two values of 1 and 0 , where 1 represents nodes that "selected as MPR" and 0 represents nodes that "not selected as MPR". As shown in Figure 8 sensor_3 not selected as MPR and sensor_4 selected as MPR.

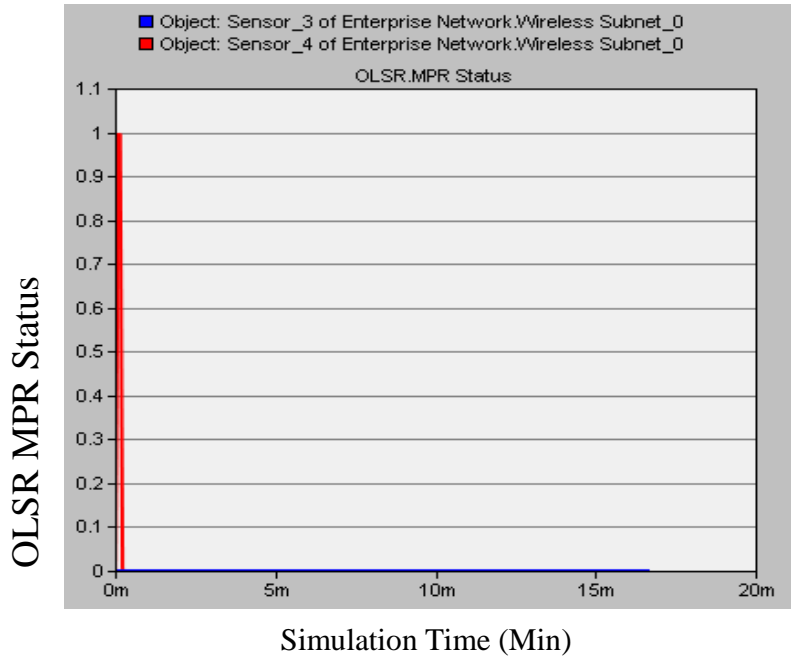

Figure 8: OLSR multi point relay status

\subsection{Route Discovery Time}

The time to discover a route to a specific destination defined as the time when a route request was sent out to discover a route to specific destination until the time a route reply is received with a route to that destination. The statistics has shown in figure 9 (a), (b) represent the time to discover a route to a specific destination by all nodes in the network. In dynamic source routing protocol, the route discovery time is constant around the mark 11.8s. It is constant as it already has the route marked out in the route cache but compared with AODV protocol, the route discovery time is minimal and varying between $0.01 \mathrm{~s}$ and $0.027 \mathrm{~s}$. However, it shows that AODV is a faster protocol at finding the route due to using one route.

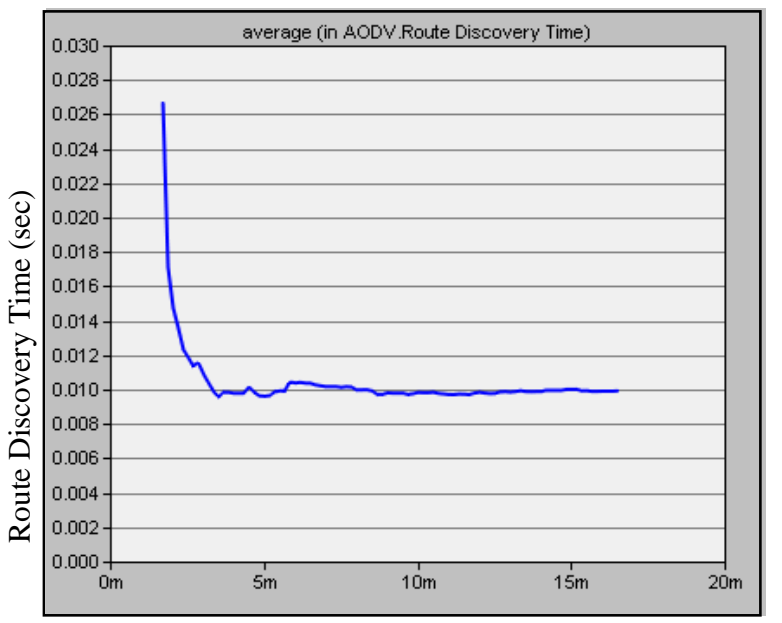

Simulation Time in AODV (Min)

Figure 9: (a) Route discovery time for AODV protocol 


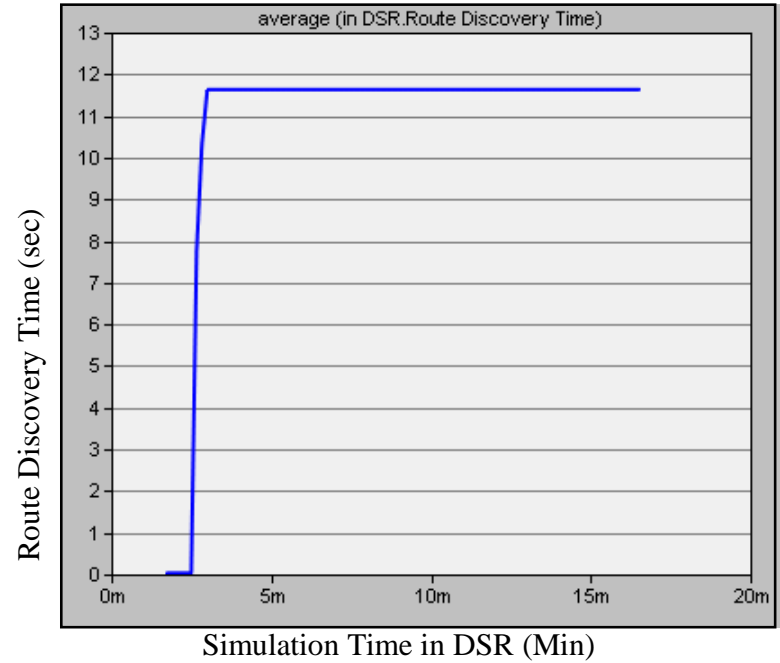

Figure 9: (b) Route discovery time for DSR protocol

\section{CONCLUSION}

In this paper, we present a comparative study with review of three routing protocols (AODV, DSR, and OLSR) that applied to nodes having random waypoint mobility characteristic. This study would be helpful in identifying the characteristics of the routing protocols and in design of radiation monitoring system based on proposed simulation model using OPNET Modeler simulator. We analyzed each routing protocol and identified the relative strengths and weaknesses of each routing protocol. The results show that OLSR achieved lower delay and routing overhead than both AODV and DSR protocols. Also, AODV protocol is faster to discover the route than DSR protocol that dropped fewer packets and caused fewer total loads than AODV protocol. In addition to, we observed that when we increased the number of nodes in the simulation model scenarios to double, the behavior of simulated protocols was not impacted by the slight change of obtained results. In the future work, we will use the obtained results from studying of different routing protocols and algorithms to implement reliable networked control systems.

\section{REFERENCES}

[1] Adel.S.El ashheb, "Performance Evaluation of AODV and DSDV Routing Protocol in Wireless sensor network Environment", International Conference on Computer Networks and Communication Systems, IPCSIT vol.35, Singapore, 2012.

[2] C. E. Perkins, E. M. Royer, S. R. Das and Mahesh K. Marina, "Performance Comparison of Two On-Demand Routing Protocols for Ad Hoc Networks", IEEE Personal Communications, February 2001,pp.16-18.

[3] Fan Bai and Ahmed Helmy, "A Survey of Mobility Models in Wireless Ad hoc Networks", chapter1 in Wireless Ad-Hoc Networks, Kluwer Academic, 2006.
[4] S.Koteswararao, M.Sailaja, T.Madhu, "Performance Evaluation of Wireless Sensor Network Routing Protocols for Real Time Application Support", Volume 11 Issue 20 Version 1.0 December 2011.

[5] Mrunali Dhande, C. G. Dethe, "Performance Evaluation of Various Routing Protocols in Wireless Sensor Networks", International Journal of Engineering Research and Applications, April 2014.

[6] Ali Abdul-Majed, Hasein Issa, "Performance Evaluation of Dynamic Source Routing Protocol (DSR) on WSN", International Journal of Computing and Digital Systems, 2012.

[7] Madhav Sharma, Rajeshwar Dua, Vijay Mohan Shrimal, " Comparison of Different Routing Protocols (DSR \& AODV) on Behalf of Evaluation of Different Routing Parameters With Constraints", International Journal of Computer Networks and Wireless Communications, June 2012.

[8] Hussein Mohammed Salman, "Survey of routing protocols in wireless sensor networks", International Journal of Sensors and Sensor Networks, Jan 2014.

[9] Jun Zheng, Abbas Jamalipour, "Wireless sensor networks: a networking perspective", John Wiley \& Sons, 2009, ch1, ch2.

[10] Waltenegus Dargie, Christian Poellabauer, "Fundamental of wireless sensor networks Theory and Practice", Wiley Series on Wireless Communications and Mobile Computing, first edition, 2010, ch.7, pp.173-180.

[11] C. E. Perkins and E. M. Royer, "Ad Hoc On-demand Distance Vector Routing", Proc. 2nd IEEE Wksp. Mobile Comp. Sys. and Apps., Feb.1999, pp. 90-100.

[12] D. Johnson and D. Maltz, "Dynamic Source Routing in Ad Hoc Wireless Networks", T. Imielinski and H. Korth, Eds. Mobile Computing, Ch. 5, Kluwer, 1996.

[13] Muhammad Ullah, Waqar Ahmad, "Evaluation of Routing Protocols in Wireless Sensor Networks", Master's Degree Thesis, karlskrona May 2009.

[14] The OPNET Modeler Simulator, available, "http://www.opnet.com"

[15] Asar Ali, Zeeshan Akbar, "Evaluation of AODV and DSR Routing Protocols of Wireless Sensor Networks for Monitoring Applications", Master's Degree Thesis, karlskrona October 2009.

[16] Ahmed M. Abbas , Abdelhalim Zekry , Imbaby I. Mahmoud, and Hussein A. Elsayed, "An SDL Design and Implementation of a Radiation Monitoring Network Using WCDMA", Journal of Communication and Computer, David publishing, 2012. 